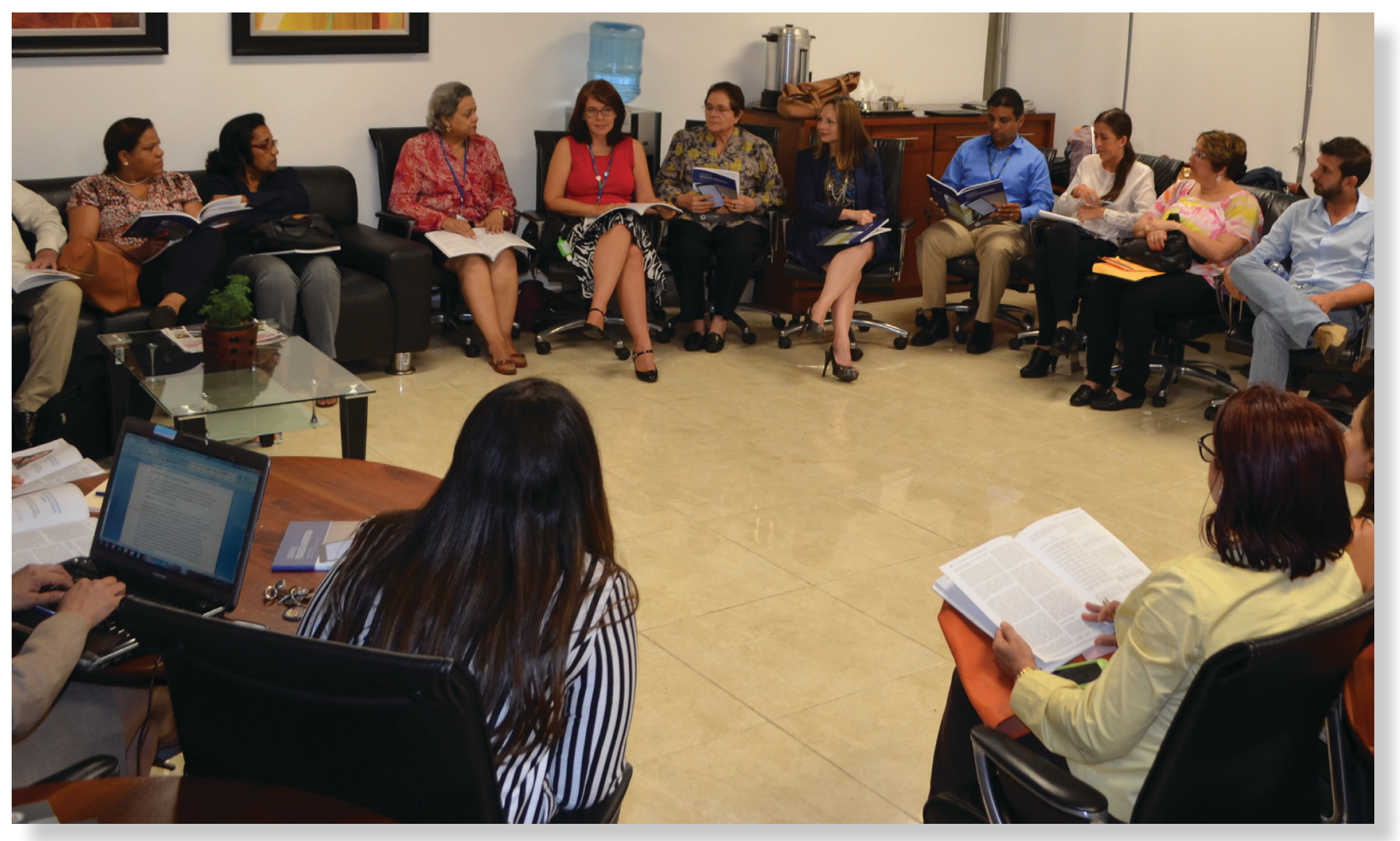

\title{
La XIV peña pedagógica
}

Estos encuentros, de carácter informal entre el profesorado de la PUCMM, se organizan dos veces al año, para compartir ideas y opiniones de los artículos publicados en la última edición del Cuaderno de Pedagogía Universitaria. La más reciente se llevó a cabo el miércoles 15 abril de 2015, en el campus Santiago. A continuación reseñamos las ideas principales del encuentro.

I encuentro inició con las palabras de bienvenida de la directora del Centro de Desarrollo Académico (CDAC), Claudette García, quien se dirigió a los presentes, en nombre del Consejo editorial del Cuaderno de Pedagogía Universitaria, aprovechando la ocasión para informar a la comunidad académica sobre las novedades en la publicación.

Se realizó el lanzamiento oficial del Cuaderno en versión digital, bajo los lineamientos del software Open Journals Systems (OJS), uno de los programas de código libre más utilizados por instituciones académicas y de investigación para la gestión editorial de revistas en línea. Esta nueva versión en línea se convierte en un producto cualitativamente diferente a la versión impresa, pues responde a la incorporación de criterios de calidad editorial reconocidos internacionalmente, que permiten su aceptación en diversos servicios de indización y hemerotecas virtuales de acceso abierto.

Además, se anunció que a raíz de los cambios en los criterios de calidad editorial, se creó un Comité asesor como un órgano del Consejo editorial, que se encarga de velar por los parámetros de calidad científica de la revista y además, le concierne proponer y asesorar la labor del Consejo editorial. Este Comité asesor lo integran la Vicerrectora Académica de Grado, Rafaela Carrasco; la profesora Ana Margarita Haché y el profesor Miguel Zabalza. 
Una vez anunciados los cambios la directora del CDAC dio paso a la articulista Niris Caba, quien compartió con los presentes el artículo de su autoría junto a Raúl Yunén, titulado "Estrategias de lectura ¿moda o necesidad académica?" Manifestó que el artículo se deriva del Proyecto Pedagógico de la Especialidad en Pedagogía Universitaria. Explicó que antes de elegir el tema hicieron un levantamiento de información entre docentes y estudiantes del Departamento de Comunicación Social e identificaron que existía una problemática con relación a la lectura y la argumentación, habilidades que son transversales para los comunicadores.

Expresó que a lo largo de la investigación fueron madurando en sus estrategias y sentían que los estudiantes también lo hacían con ellos. Las principales estrategias utilizadas fueron resumir para uno mismo y leer con ayuda de guías. Explicó que al principio los estudiantes sentían que tener en cuenta los criterios proporcionados por el profesor les limitaba, pero que cuando recibían los resultados de las evaluaciones y se percataban de que les ayudaba, su actitud cambiaba. Indicó que lo importante, en el caso de resumir para uno mismo, es que no iban de la lectura al examen, sino que estaba mediado por la escritura del resumen. Al final tuvieron una curva de aprendizaje satisfactoria, según la articulista.

En el caso de leer con ayuda de guías realizaron tres actividades, primero asignaron una guía con inferencias, luego preguntas de macro estructura y el uso autónomo de inferencias. La articulista compartió con los presentes sobre la creación de un grupo en Facebook, que sirvió como motivación para los estudiantes, quienes lo utilizaron como un espacio de comunicación colectiva y que les sirvió mucho para el aprendizaje, según sus propios comentarios.

Las estrategias condujeron a agregar un componente lingüístico a lo largo de la carrera que permita hacer placentera la lectura académica. Generalmente se parte de la premisa de que a los estudiantes no les gusta leer, pero no es así; lo importante no es solo que lean, sino que comprendan lo leído. Además, agregó que sus estudiantes se motivan más cuando encuentran un sentido práctico en la lectura y la escritura, como por ejemplo la redacción de su investigación final.

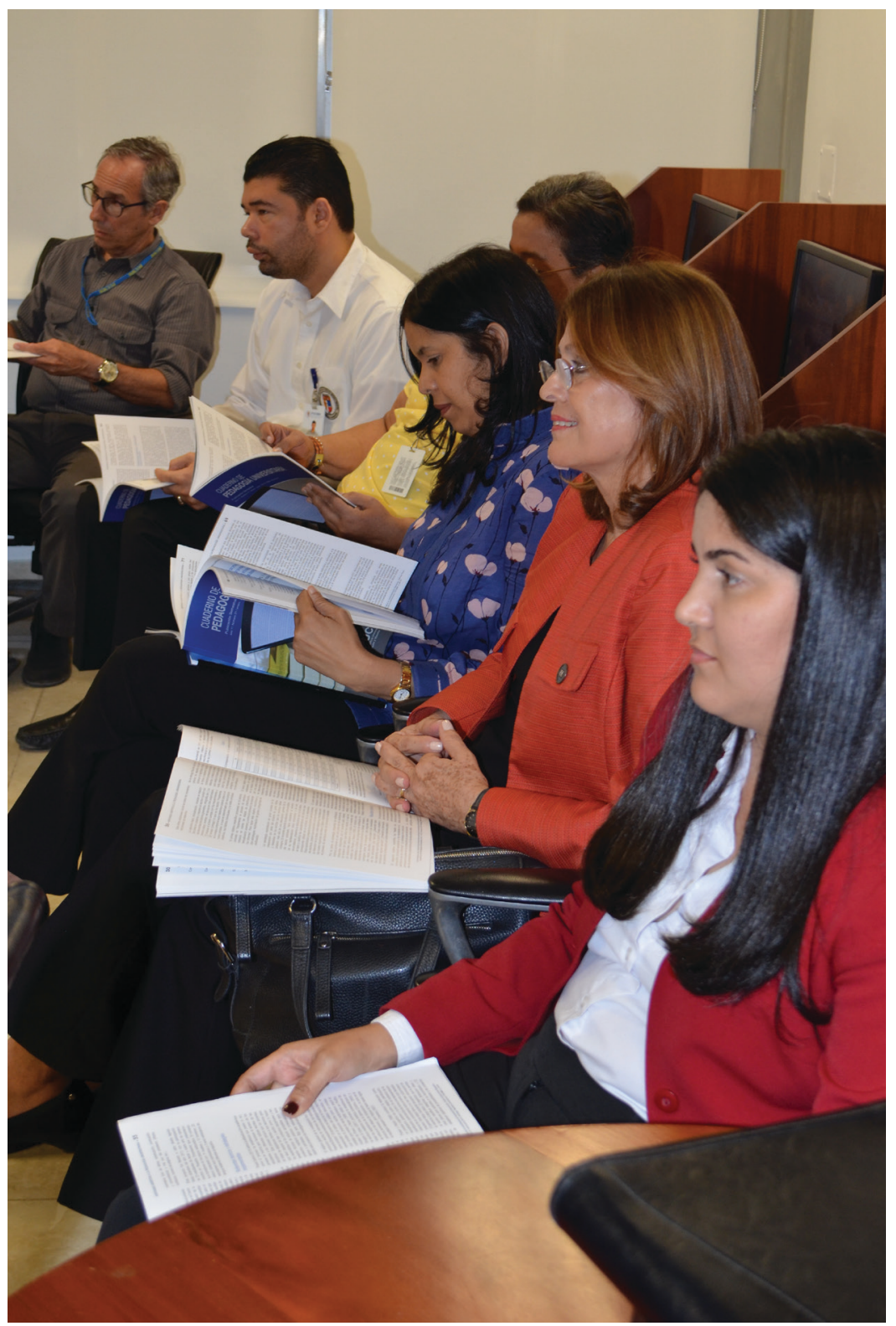

El profesor Ariosto Díaz expresó que la lectura desarrolla la imaginación y que es un hábito que debe desarrollarse desde niños, por lo que no se puede esperar a llegar a la universidad para desarrollarlo. Asintió con la articulista explicando que sus estudiantes leen si les interesa.

La profesora Mary Abreu señaló que es una responsabilidad compartida. El deber del profesor es buscar estrategias para motivar, pero el estudiante es responsable de motivarse a aprender. En este sentido, Mayra Ruiz, profesora del Departamento de Comunicación Social, comentó que muchos de sus estudiantes no tienen hábito de lectura y que para motivarles, en este período, les asignó la lectura del libro "Los siete hábitos de la gente altamente efectiva", tuvieron que resumirlo y exponerlo y se mostraron bastante motivados.

Yenny Rosario motivó a los profesores presentes a cuestionarse sobre las preguntas que se hacen a los estudiantes a partir de las lecturas que se asigna. 
La articulista Niris Caba hizo hincapié en la parte de la retroalimentación; aseguró que cuando se corrige al estudiante en el error y se le proporcionan estrategias para mejorar se producen resultados positivos.

La profesora Carmen García cuestionó a la autora acerca de los resultados de la investigación en su práctica docente, a lo que la articulista respondió que el proceso de investigación la llevó a comprender que los estudiantes no suelen valorar las estrategias al principio, sino cuando logran experimentar los cambios que generan en su proceso de aprendizaje.

Antonio Rivero señaló que en la asignatura de matemáticas es mejor para los estudiantes aprender los conceptos fundamentales, para que a partir de los mismos puedan generar otros conocimientos. Comentó que en su experiencia, las retroalimentaciones evidencian que hay una comprensión de que es lo que tienen que hacer y que al final estas estrategias permiten que tengan aprendizajes significativos.

La profesora Cristina Nuñez cuestionó a la autora sobre la reacción de los estudiantes ante la nueva metodología implementada. La articulista manifestó que se aplicó un cuestionario de valoración en ambas asignaturas y que las evaluaciones señalaron que habían encontrado datos diferentes en textos que antes leían, sentían la libertad de expresar sus comentarios y que apreciaron la parte de la retroalimentación como estrategia para la incorporación de nuevos aprendizajes.

Otro de los artículos comentados durante La peña fue el de la articulista Sonja Arias, quien explicó que su investigación se originó en el contexto de la asignatura Pasantía Empresarial, la cual se imparte en el tercer año de la carrera de Administración de Empresas. Señaló que la problemática sobre la cual trabajó fue la baja calidad de los escritos de los estudiantes y se enfocó en mejorar las producciones escritas.

La articulista compartió con los presentes que antes de realizar el Diplomado en Lectura y Escritura les asignaba a sus estudiantes un informe final de pasantía, que marcaba y corregía con muchos comentarios; sin embargo, le quedaba la duda de que los estudiantes comprendieran sus recomendaciones, por lo que llegó a la conclusión de que un producto terminado no ayudaba al estudiante a mejorar sus habilidades de escritura, si no se aseguraba la retroalimentación.

Refirió que el Diplomado la ayudó a seleccionar mejor las estrategias para corregir esta situación. Lo primero que hizo fue realizar guías claras y con detalles claros para los estudiantes. Señaló que en esta materia no hay un horario de clases asignado, pero que se reunían periódicamente para mejorar el escrito y luego se enviaban por la plataforma virtual de la Universidad.

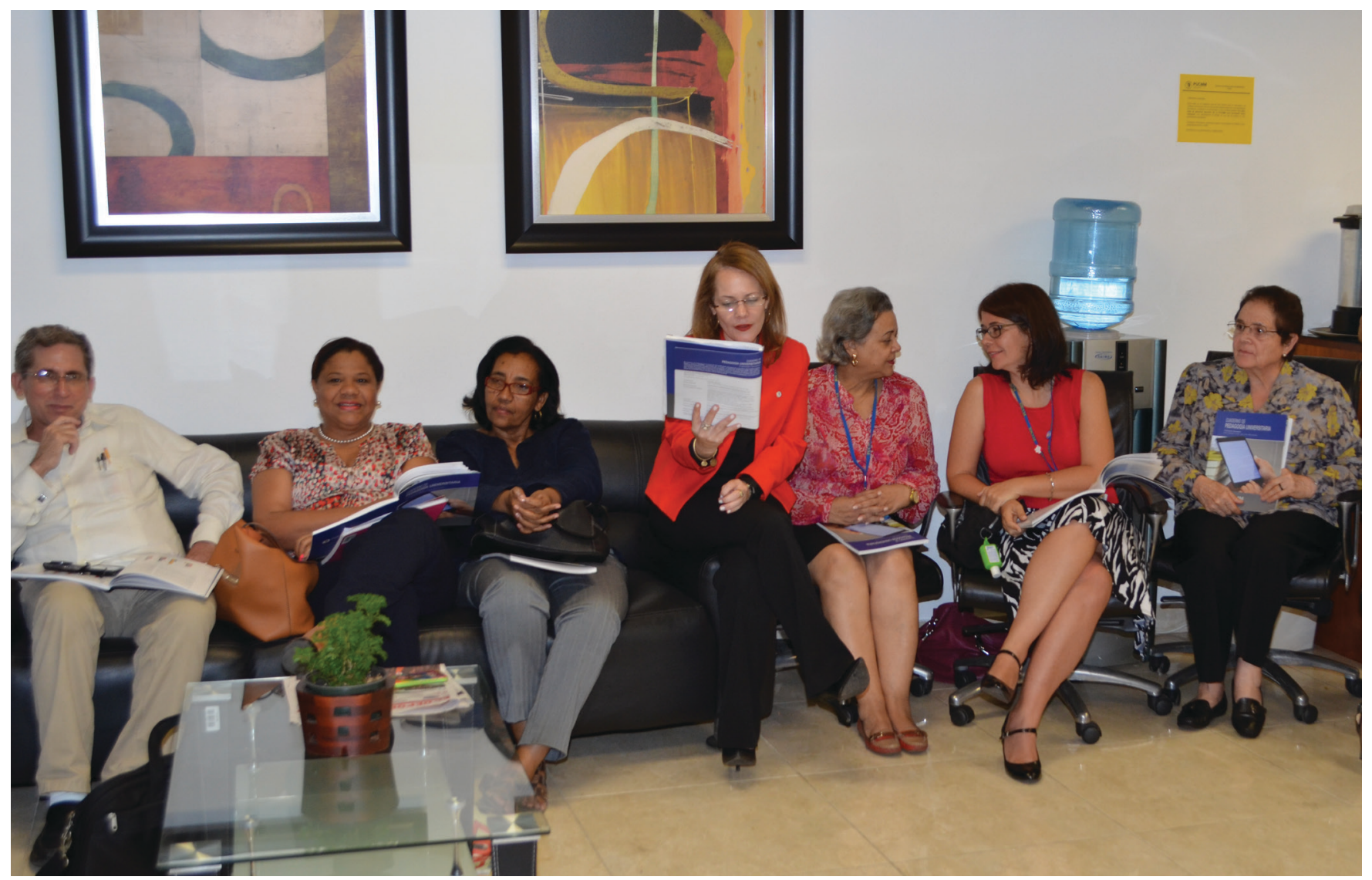


Las estrategias utilizadas fueron un cuestionario de verificación, entrevistas, reuniones para el monitoreo de los avances, revisiones en Word, retroalimentación, corrección de pares y una rúbrica de evaluación. Esas estrategias permitieron asegurar el aprendizaje para las cuales fueron seleccionadas, considerando como una de las más significativas, por la motivación que propició al estudiantado, la retroalimentación.

Arias comentó que gracias a estas intervenciones los estudiantes mejoraron considerablemente sus escritos. Afirmó que este proceso conlleva un esfuerzo de trabajo del docente, pero es un esfuerzo retribuido con el desarrollo y la autonomía que se genera en el estudiante.

Ylona de la Rocha expresó que la estrategia que más le gustó fue la de revisión de pares y que según su juicio los docentes no la utilizan porque creen que el estudiante va a ser condescendiente, pero no es así. Arias corroboró afirmando que si se proveen las herramientas necesarias se desarrolla la capacidad y se convierten en evaluadores críticos.

La articulista Rosario Bisonó compartió con los presentes algunas opiniones de los docentes del Departamento de Ingeniería Industrial, quienes habían identificado una problemática de escritura en los proyectos finales de los estudiantes. Afirmó que por mucho tiempo se quedaron estancados en la identificación de la problemática, pero que no se realizaba ninguna intervención. Aseguró que el Diplomado en Lectura y Escritura marcó un antes y un después en el Departamento, pues se partía del paradigma de que los ingenieros eran parcos a la hora de escribir, pero se ha comprobado que también pueden desarrollar esta competencia.

Bisonó indicó que para ella el mayor aporte que se ha hecho al Departamento después de su investigación fue poder describir criterios que se pueden emplear para evaluar la escritura y la motivación que generó a los demás docentes del Departamento. La articulista valoró el trabajo multidisciplinario que había realizado con docentes del área de Español, quienes le acompañaron y apoyaron en el proceso; motivó a los presentes a seguir este tipo de trabajo colaborativo y a tener la humidad de pedir apoyo a otros docentes cuando la necesiten. Afirmó que estos espacios pueden ayudar mucho a fortalecer nuestra comunidad académica.

Gloria Velázquez comentó que como profesora del Departamento de Nutrición y Dietética ha percibido que a los estudiantes se les dificultan explicar sus conocimientos a través de la escritura. Felicitó a los articulistas presentes y señaló que se ha motivado a realizar cambios en su metodología de evaluación a través de estas reflexiones.

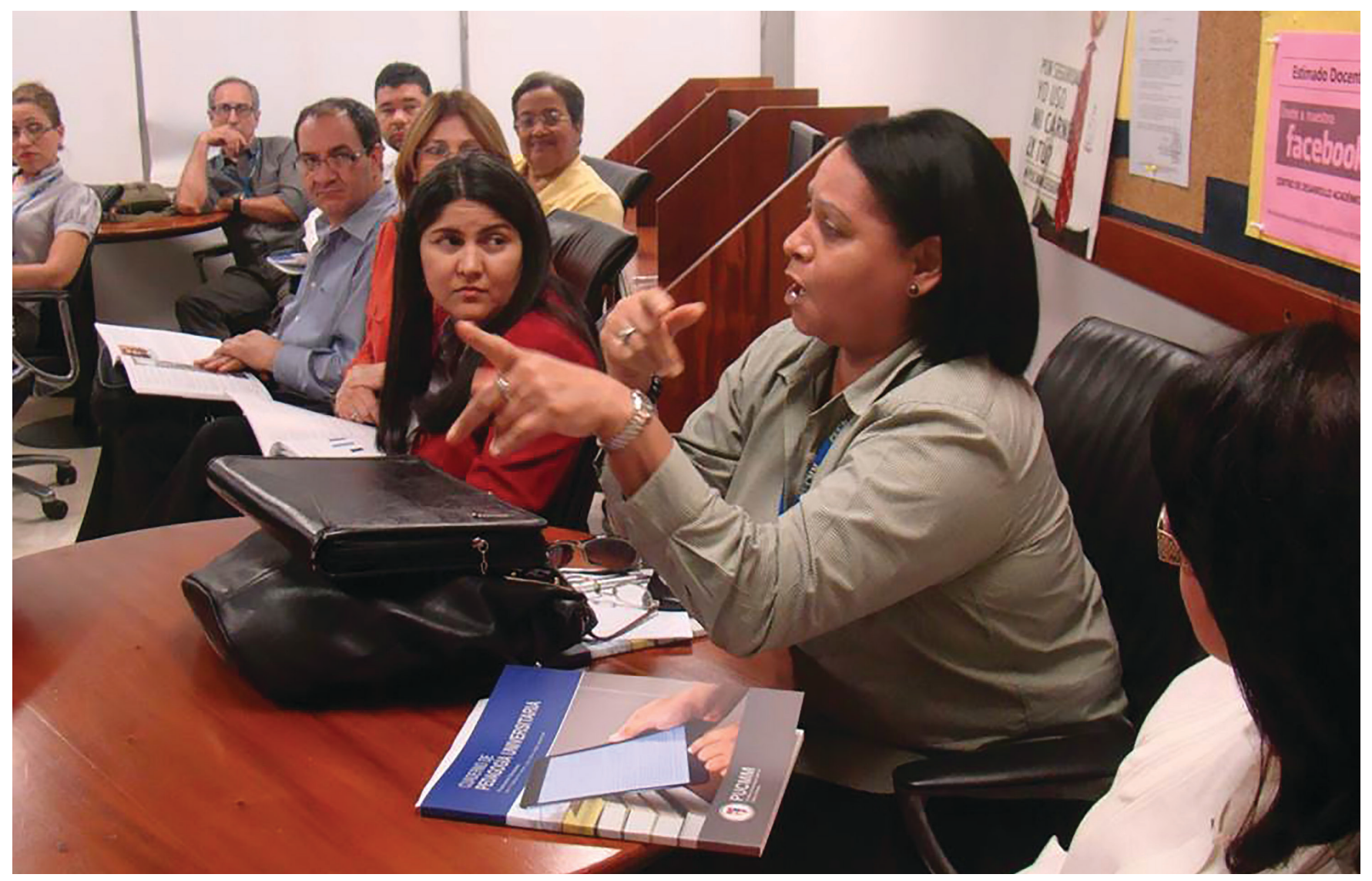


La articulista Patricia Tineo explicó que el marco de su proyecto fue la asignatura Operación de Recepción y que utilizó las estrategias de estudios de casos, juegos de roles, evaluación de pares y una rúbrica de evaluación de los aprendizajes previos. Una de las metodologías utilizadas consistió en dividir al grupo en subgrupos de cuatro a seis estudiantes para que realizaran una investigación a través de procesos y conceptos. Buscaron fuentes primarias, libros, entrevistaron ejecutivos de la industria, luego crearon su propio proceso, que defendieron con un texto argumentativo que justificaba las razones por las cuales llevaron a cabo el proceso en la forma en que lo hicieron.

Explicó que utilizó esta metodología con un grupo y que utilizó otro grupo como control, en el que no aplicó la metodología. Afirmó que quedó comprobado en la investigación que estas estrategias funcionan y que se pueden evidenciar en el artículo.

La profesora Gema González compartió que la motivación que generan los profesores para la lectura y escritura es fundamental para un proceso de enseñanza aprendizaje de calidad.

A manera de conclusión, la profesora Liliana Montenegro felicitó a los profesores. Expresó que para ella es una inmensa alegría ver que el Diplomado no solo les hace diferentes personas, sino que también impacta en su forma de dar clases y a sus departamentos.

A partir de las intervenciones afirmó que no coloca la lectura y la escritura en el centro, sino como un medio y que el centro es compartir saberes. El estudiante que llega a la Universidad llega para formarse y graduarse

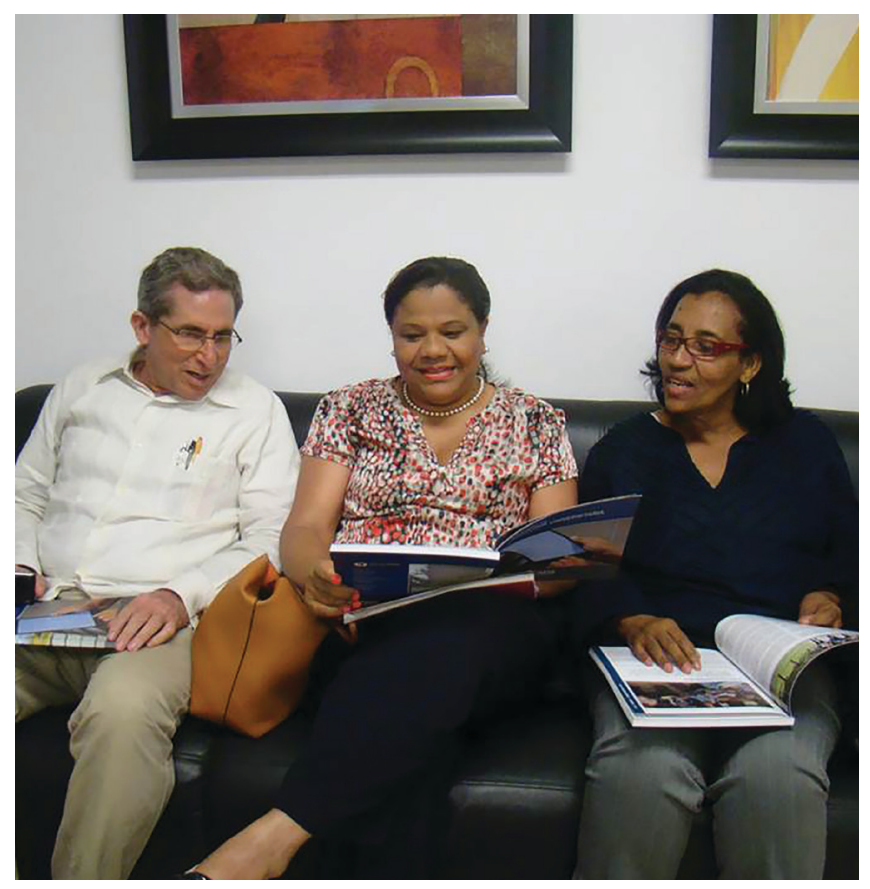

en un área disciplinar, debe utilizar la lectura y escritura como una herramienta para poder aprender y expresar lo que aprendió. Indicó que como país debemos replantear, no solo la educación, sino toda la educación cultural, como por ejemplo Cuba donde la población lee en las calles, esto así por ser una política estatal donde se abarató el libro para que sea un bien común.

Aseguró que como Universidad tenemos el poder de decisión en cómo instrumentar que los estudiantes puedan ser profesionales que dominan la lectura y la escritura y que debemos felicitar la visión de la PUCMM de crear profesionales bien formados en lectura y escritura. Testificó que esta visión ha sido fruto del arduo trabajo de muchos.

Finalmente, la directora del CDAC, Claudette García, agradeció a los presentes por su asistencia y aportes y aprovechó para motivarles a seguir la formación pedagógica a través de las ofertas formativas que el Centro ofrece para los docentes.

Asistentes a La peña: Leonardo Belliard, Mayra Ruiz, Wendy Moronta, Ariosto Díaz, Hortencia Corcino, Carmen Pérez, Miriam Valdez, Sonja Arias, Wanda Sepúlveda, Antonio Rivero, Carmen García, Liliana Montenegro, Ramón Viñas, Yenny Rosario, Patricia Tineo, Frances Grullón, Nelsa Acosta, Niris Caba, Ylona de la Rocha, Ana Margarita Haché, Cristina Núñez, Dariel Suárez, Gisela León, María Luisa Tavárez, César Payamps, Harold Paz, Steven Sánchez, Petra Velázquez, Gema González, Roberto Caballero, Arsenio Guzmán, Rosanna Crespo, Rosario Bisonó, Mary Abreu, Claudette García, Sandra Hernández, Oliva Hernando, Florangel Mata, Florilena Paredes, Giovanna Cabreja, Larissa Madera, Nora Ramírez y Yenisse Ramos.

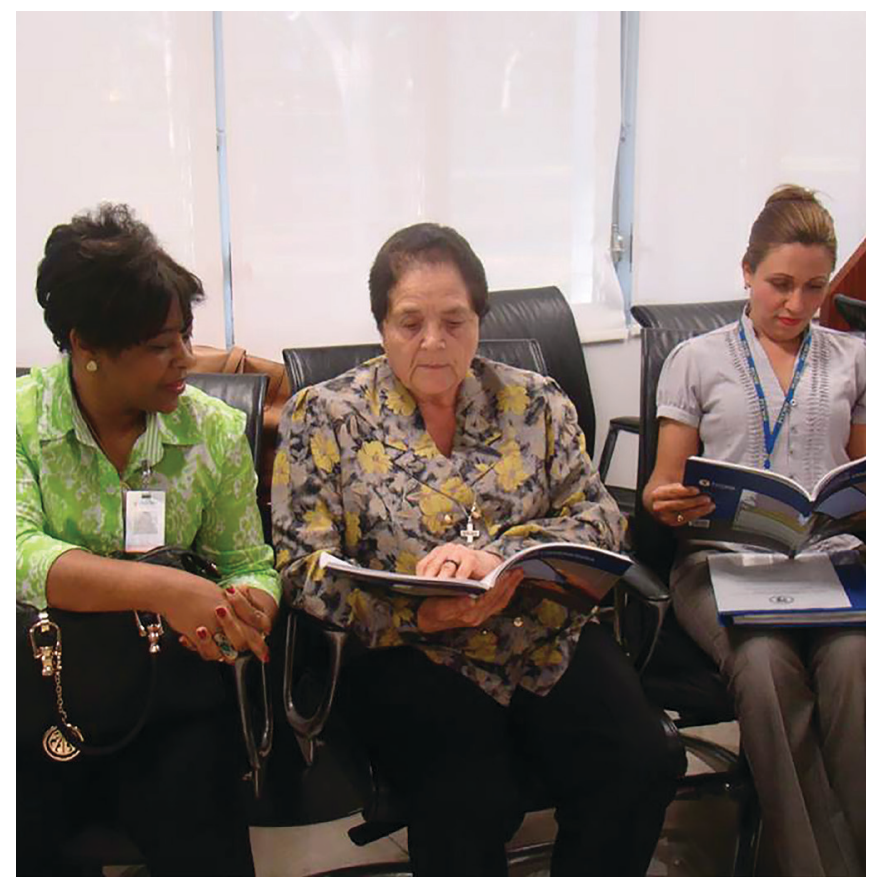

